\title{
Magnon dispersion in field-induced magnetically ordered phases
}

\author{
Masashige Matsumoto $^{\mathrm{a}, *}$, B. Normand ${ }^{\mathrm{b}}$, T.M. Rice ${ }^{\mathrm{a}}$, Manfred Sigrist $^{\mathrm{a}}$ \\ ${ }^{a}$ Theoretische Physik, ETH-Hönggerberg, Zürich CH-8093, Switzerland \\ ${ }^{\mathrm{b}}$ Départment de Physique, Université de Fribourg, Fribourg CH-1700, Switzerland
}

\begin{abstract}
The magnon dispersion in the field-induced and pressure-induced antiferromagnetically (AF) ordered phase in $\mathrm{TlCuCl}_{3}$ are studied. In the pressure-induced AF phase, there are two transverse phase (Goldstone) modes and one gapped longitudinal amplitude mode. A magnetic field lifts the degeneracy, and it gives rise to three separated magnon branches in the field-induced AF phase, where the lowest lying mode corresponds to a Goldstone mode. A model consisting of three distinct dimer sublattices is proposed to account for the plateaus which appear in magnetization curves of $\mathrm{NH}_{4} \mathrm{CuCl}_{3}$.
\end{abstract}

PACS: $75.40 . \mathrm{Gb} ; 75.30 . \mathrm{Cr} ; 75.10 .-\mathrm{b}$

Keywords: Magnon; Quantum phase transition; Field-induced magnetic order; Pressure-induced magnetic order; Magnetization plateau

$\mathrm{TlCuCl}_{3}, \mathrm{KCuCl}_{3}$, and $\mathrm{NH}_{4} \mathrm{CuCl}_{3}$ are isostructual quantum spin system consisting of two-leg ladders separated by $\mathrm{Tl}^{+}, \mathrm{K}^{+}$, and $\mathrm{NH}_{4}^{+}$ions. These compounds can be considered as coupled two-leg $S=\frac{1}{2}$ Heisenberg AF ladders, and show various types of magnetization curves. $\mathrm{NH}_{4} \mathrm{CuCl}_{3}$ has magnetization plateaus at $\frac{1}{4}$ and $\frac{3}{4}$ of the saturation moment, while $\mathrm{TlCuCl}_{3}$ and $\mathrm{KCuCl}_{3}$ have no plateaus in their magnetization curves [1]. $\mathrm{TlCuCl}_{3}$ has a finite critical field, $H_{\mathrm{c}}=5.7 \mathrm{~T}$. For $H>$ $H_{\mathrm{c}}, \mathrm{AF}$ order perpendicular to the field appears simultaneously with the uniform magnetization [2]. This magnetic field-induced order can be interpreted as a condensation of the lowest lying magnon mode driven soft by the magnetic field [3-6]. Oosawa et al. found the same AF spin structure in $\mathrm{TlCuCl}_{3}$ under hydrostatic pressure, $P=1.48 \mathrm{GPa}$ [7]. Pressure increases interdimer interactions, leading to a wider magnon band, and so can collapse the excitation energy gap, $0.7 \mathrm{meV}$ for

\footnotetext{
*Corresponding author. Tel.: +41-1-633-3175; fax: +41-1633-1708.

E-mail address: spmmatu@itp.phys.ethz.ch (Masashige Matsumoto).
}

$\mathrm{TlCuCl}_{3}$. The bond-operator formulation used in our previous work [6] is applied to the field and pressureinduced orderings. In this work, we simply assume that interladder interactions increase linearly with pressure.

In the pressure-induced AF phase, there are two-fold degenerate phase excitation modes and one amplitude mode as in Fig. 1(a), since both directions perpendicular to the induced staggered moment are equivalent. An external magnetic field lifts this degeneracy and gives rise to three distinct magnon branches in the field-induced phase [see Fig. 1(b)]. In the pressure-induced AF phase, we estimate the excitation gap of the amplitude mode is around $5 \mathrm{meV}$ under $P=1.48 \mathrm{GPa}$, and a staggered moment of $60 \%$ of the saturation moment. The amplitude mode should be observable in inelastic neutron scattering experiments.

Very recently, Oosawa et al. found two almost nondispersive magnon branches at 1.8 and $3 \mathrm{meV}$ in $\mathrm{ND}_{4} \mathrm{CuCl}_{3}$ [8]. In addition to this, we can expect another low lying branch at around $0.3 \mathrm{meV}$, since there is AF order already at zero field [9]. These results imply that there are three weakly interacting distinct dimer sublattices, A, B, C, whose intradimer interactions 

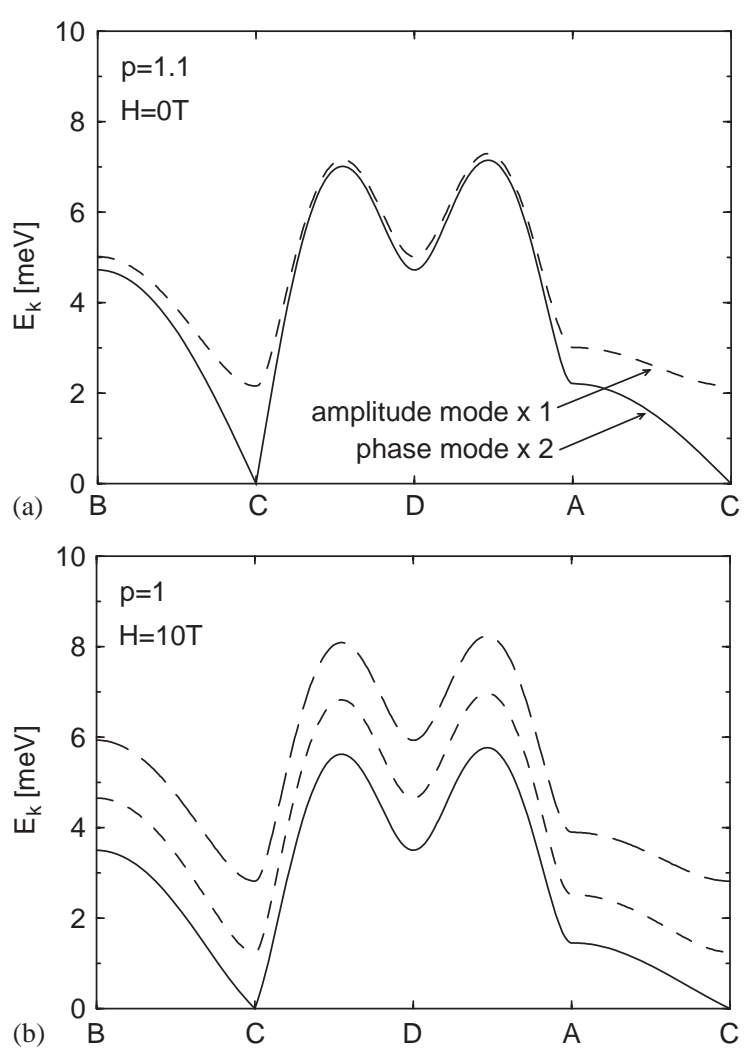

Fig. 1. Magnon dispersions in $\mathrm{TlCuCl}_{3}$ for (a) pressure-induced AF phase, (b) field-induced AF phase. We have used the same exchange parameters in our previous work [6]. For (a), interladder interactions are increased by a factor of $\lambda(p)$ which expresses pressure in our model. The $x$-axis labels represent the reciprocal-space points $\mathrm{B}=(0,2 \pi, 2 \pi), \quad \mathrm{C}=(0,0,2 \pi), \quad \mathrm{D}=$ $(0,0,0), \mathrm{A}=(\pi, 0,0)$.

are characterized by $0.3,1.8,3 \mathrm{meV}$, respectively. Motivated by these results, we propose a model consisting of three distinct dimer sublattices whose volume fractions are $\frac{1}{4}, \frac{1}{2}, \frac{1}{4}$, respectively, to reproduce the magnetization plateaus at $\frac{1}{4}$ and $\frac{3}{4}$ of the saturated moment. The details will be published elsewhere [10]. As we can see in Fig. 2, there are three ordered phases, I, II, and III, where staggered moments are induced by A, B, and $\mathrm{C}$ dimer sublattices, respectively. This is consistent with the phase diagram obtained by specific heat and high-field magnetization measurements [9]. The uniform magnetization curve, $M_{\mathrm{z}}$, reproduces the plateaus at $\frac{1}{4}$

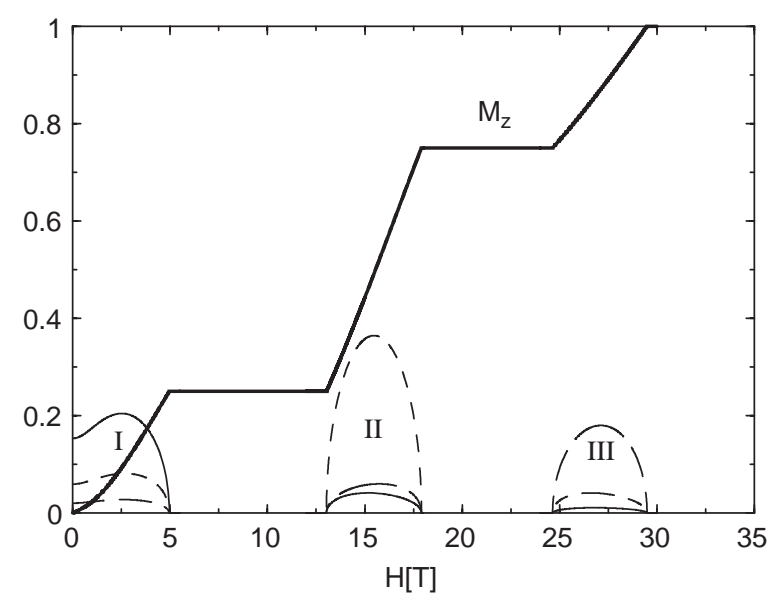

Fig. 2. Normalized magnetization curves per volume. Thick solid line, $M_{\mathrm{z}}$, is uniform magnetization. Thin solid line, dashed line, and long dashed line are staggered magnetization for A, B, and $\mathrm{C}$ dimer sublattices, respectively.

and $\frac{3}{4}$ of the saturated moment. We can predict that these plateaus disappear under high pressure which increases interdimer interactions between the three distinct dimer sublattices.

We would like to thank A. Oosawa, Ch. Rüegg, and H. Tanaka for valuable discussions on recent experimental results. This work is supported by the Japanese Society for the Promotion of Science (JSPS) and the MaNEP program of the Swiss National Fund.

\section{References}

[1] W. Shiramura, et al., J. Phys. Soc. Jpn. 66 (1997) 1900; W. Shiramura, et al., J. Phys. Soc. Jpn. 67 (1998) 1548.

[2] H. Tanaka, et al., J. Phys. Soc. Jpn. 70 (2001) 939.

[3] T. Kato, et al., J. Phys. Soc. Jpn. 67 (1998) 752.

[4] Ch. Rüegg, et al., Appl. Phys. A 74 (2002) S840; Ch. Rüegg, et al., Nature (London) 423 (2003) 62.

[5] T. Nikuni, et al., Phys. Rev. Lett. 84 (2000) 5868.

[6] M. Matsumoto, et al., Phys. Rev. Lett. 89 (2002) 077203.

[7] A. Oosawa, et al., J. Phys. Soc. Jpn. 72 (2003) 1026.

[8] A. Oosawa, et al., preprint, cond-mat/0304172.

[9] B. Kurniawan, et al., J. Phys.: Condens. Matter 11 (1999) 9073.

[10] M. Matsumoto, Phys. Rev. B 68 (2003) 180403(R). 